\title{
Using a computer simulation of three slot machines to investigate a gambler's preference among varying densities of near-miss alternatives
}

\author{
Otтo H. MacLin \\ University of Northern Iowa, Cedar Falls, Iowa \\ MARK R. Dixon \\ Southern Illinois University, Carbondale, Illinois \\ DUSTIN DAUGHERTY \\ University of Northern Iowa, Cedar Falls, Iowa \\ AND \\ STacey L. Small \\ Southern Illinois University, Carbondale, Illinois
}

\begin{abstract}
The present article describes a software program in Visual Basic .NET designed to simulate three slot machines on a computer screen. This software is described in detail regarding utility, downloading, and usage; and data are presented illustrating the software's potential for researchers interested in gambling behavior. A simulation of multiple slot machines such as this enables researchers to evaluate players' preferences across various machines. In the highlighted experiment, 18 recreational slot machine players played the software for extra course credit and a chance at cash prizes. All participants played a version of the simulation in which every 5 th response on average was a win, whereas the remaining trials were a loss. However, on those loss trials, a varying distribution of almost wins or near misses (i.e., two winning symbols on the payoff line and the final winning symbol directly above or below the payoff line) were presented in percentages of 15,30 , or 45 . While no preferences across the three options could be predicted on the basis of reinforcement history alone, deviations from equal choices across the games were noted and appeared to be the result of the presentations of near-miss losing trials. Implications for a greater understanding of pathological gambling are presented.
\end{abstract}

Legalized gambling has risen in popularity across the United States over the past 20 or so years. In 1988, only two states, Nevada and New Jersey (Ghezzi, Lyons, \& Dixon, 2000), allowed legalized casino gambling; today, however, all states except Utah and Hawaii have some form of legalized gambling. Also, anyone with an Internet connection has access to various forms of gambling at the click of a mouse. The consequences of increased opportunities to gamble include increased revenue for struggling state economies, new jobs for unemployed workers, and tax dollars for education initiatives (Dixon \& Moore, 2006). There is a darker side to the rise in legalized gambling, however: the growing number of people suffering from the impulse control disorder of pathological gambling. Whereas they once accounted for approximately $1 \%$ of the population, today it is estimated that problem or pathological gamblers make up almost $3 \%$ of the population (Shaffer, Hall, \& Vander Bilt, 1999). While the increase in pathology may be only correlated and not a direct result of greater gambling opportunities, the time is right for psychological researchers interested in gambling to begin rigorous lines of inquiry. Basic human behavioral processes need to be understood and clinical treatments attempted. With Gambler's Anonymous apparently yielding a pathetic $8 \%$ success rate after 1 year (Stewart \& Brown, 1988), the scientific community has a great opportunity to shed empirical light on a growing psychological disorder.

Perhaps a first step in understanding the behavior of a gambler is to evaluate his behavior in ways that are not feasible in an actual casino. Such a method was described by MacLin, Dixon, and Hayes (1999) in a software announcement describing a computerized simulation of a slot machine that allowed for the collection of various forms of behavioral data. The simulation allowed for altering both the probability of wins and losses, and the various types of slot machine reel symbols that would eventually land on the payoff line. The experimenter could also con-

M. R. Dixon, mdixon@siu.edu 
trol the size of each payoff and the number of credits that could be wagered by a subject at any time. The MacLin et al. (1999) simulation allowed researchers interested in gambling to ask research questions and to manipulate variables in a way that would be impossible with a real slot machine in a real casino, due to federal regulations on game randomness (Dixon \& Delaney, 2006).

Following the publication of the MacLin et al. (1999) software, the programming language used in its design (Microsoft Visual Basic 6.0) underwent a significant overhaul. The new version, Visual Basic .NET, was a significant upgrade that allowed for greater programming flexibility than had previous versions. The MacLin et al. (1999) paper suffered from a major research shortcoming - an inability to control the outcome of the slot machine display trial by trial. Instead, researchers could merely select probability levels, not actual specific strings of wins and losses. In response to the newest version of Visual Basic and a need for greater control over specific trial outcomes, MacLin, Dixon, Robinson, and Daugherty (2006) designed an updated slot machine using Visual Basic .NET that also allowed for the creation of an input file containing trial-bytrial information which could be read into the simulator to display any trial sequence and jackpot size specified by a researcher. The increased flexibility of this newer version of the slot machine simulator allowed for more research questions to be asked, and it also increased the level of experimenter control during inquiry.

The behavior of a gambler on a single slot machine is interesting to examine, and gains have been made toward understanding why a gambler would continue to play a slot machine when faced with repeated losses. In a recent study by Dixon and Schreiber (2004), slot machine players on a single slot machine were asked to rate on a Likert scale from 1 to 10 how close they thought each of their losing trials had come to winning. Surprisingly, all players rated these losses quite differently on the basis of what the actual loss looked like. When a loss consisted of three losing symbols on the payoff line, it was rated low, but when it consisted of two winning symbols on the payoff line and a third symbol above or below the payoff line (on a three-reel slot machine), it was rated relatively higher. This observation of behavior has been noted before as a near-miss effect (Dixon \& Delaney, 2006), which occurs when players react differently to near misses (i.e., two winning symbols on a payoff line and a third symbol above or below the payoff line) than to total losses.

However, to investigate the near-miss effect, or any other form of gambling behavior, on a single slot machine would appear to restrict the scope of research, given the opportunities for playing slot machines that gamblers have in casinos across the country. In such casinos, many different kinds of slot machines are available to the individual gambler who, moreover, may allocate his responses across any combination of these slot alternatives in exponentially varied ways. Thus, if researchers are going to attempt an explanation of slot machine players' behavior within a naturalistic context, it would appear logical to investigate that behavior using an experimental apparatus more closely approximating reality.
The purpose of the present article, therefore, is twofold: to describe a software simulation on a computer screen of three slot machines that can each be altered in various ways by the experimenter; and, by illustrating the utility of such a simulation, to gain a better understanding of the near-miss effect.

\section{SOFTWARE OPERATION}

The slot machine simulation was written in Visual Basic .NET 2002 for any IBM compatible computer running the Microsoft Windows XP operating system. Visual Basic .NET is a graphics-oriented software program which is part of Microsoft's developer suite Visual Studio .NET. While the .NET software platform is significantly more cumbersome than machine language or the $\mathrm{C}$ programming language, it makes up for these difficulties through its easy access to the Windows file libraries that make it possible to incorporate Media Player and file sharing accessibility. The previous versions of slot machine simulators described by MacLin et al. (1999; Dixon, MacLin, \& Daugherty, 2006) allowed the user to use the software without purchasing the Visual Basic software itself, but the present simulator described in this program requires Visual Basic .NET to run properly; however, with the steadily declining cost of the software (the approximate current retail price is $\$ 100$ ), many researchers will find the expense quite modest, given the potential.

The experimenter can control various aspects of the software simulator through a graphical interface that allows for a prespecification of the percentage of trials that will be wins, losses, and near misses for each of the three slot machines. Using drop-down menus, the experimenter can also select the number of credits to be won by the participant on any given trial, as well as the amount of credits that each player will start with. To ensure experimental control for response bias toward the "left," "middle," or "right" of the slot machine, each machine can be randomized across trials, or color coded. Finally, for a given experimental session, the experimenter can specify the number of trials required and the number of optional trials to which the player will be exposed.

Figure 1 displays the graphical interface visible to the participant during the experiment. As can be seen in this figure, the participant is presented with three slot machine simulations, any one of which can be selected with a click of the "Spin" button located immediately below the respective display. The machine then displays the spinning of the reels for approximately $3 \mathrm{sec}$ and the resulting display is visible until the participant initiates the next trial. Upon a winning display, an audio media file of coins clanking in a slot machine tray is played for $1 \mathrm{sec}$.

\section{METHOD}

\section{Participants, Apparatus, and Setting}

Eighteen undergraduate psychology students at the University of Northern Iowa (age range, 18-27) participated in the experiment for extra course credit and the opportunity to win a cash prize of $\$ 10$ for scoring the highest number of points on the simulation among groups of 4 or 5 participants. Eleven participants were women. All participants were screened for gambling problems using the South 


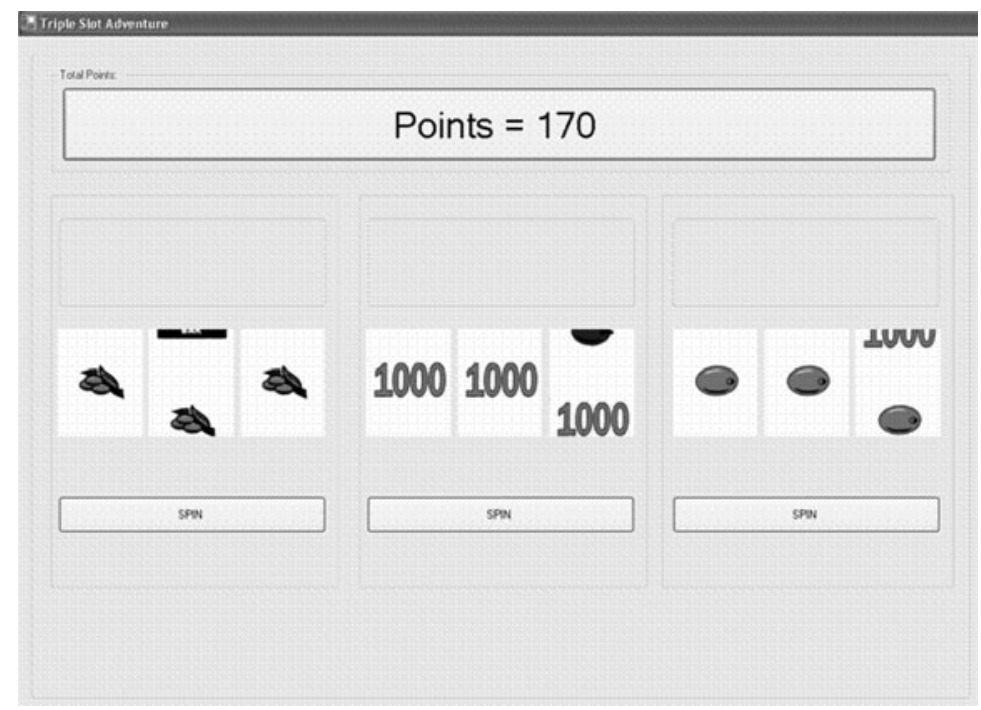

Figure 1. Graphical representation of the multislot machine interface.

Oaks Gambling Screen (Lesieur \& Blume, 1987) and scored as nonpathological gamblers. However, all participants noted that they occasionally did play slot machines on a recreational basis.

The experiment was run in the psychology department in a variety of small $10 \times 10 \mathrm{ft}$ rooms equipped with microcomputers, office equipment, and detailed computer software. Each computer contained the Microsoft Windows XP operating system, Visual Basic .NET computer programming software, and a copy of the slot machine simulation highlighted in this article.

The simulation was arranged by the experimenter with the following parameters: (1) required trials to be played, 100; (2) optional trials to be played, endless; (3) starting credits, 200; (4) cost per spin, 10 credits; and (5) credits per win, 100. Win, loss, and near-miss parameters were also preselected by the experimenter, with the following parameters: (1) wins, $20 \%$ across all machines; (2) near-misses, varied across each machine as $15 \%, 30 \%$, or $45 \%$; and (3) total losses, remaining respective percentages.

\section{Procedure}

After completing an informed consent, all participants were instructed to play the present three slot machines, in any order, and to try to earn as many points as possible. They were also told that the high score for their participant group (4-5 participants) would win an additional $\$ 10$.

Phase 1: Preextinction. All participants played the previously described multiple slot machine simulation for 100 trials under the contingencies preselected by the experimenter. In summary, all slot machines resulted in a win of 100 credits on the average of every 5th spin (i.e., $20 \%$ of the time); the cost of each spin was 10 credits. The only difference between the three games during this phase was that losses varied in physical characteristics. Each slot machine displayed the respective percentage of losses as near misses $(15 \%$, $30 \%$, or $45 \%$ of the trials). This phase was used to examine preference across the various slot displays when all reinforcing consequences were in fact equal.

Phase 2: Extinction. At the onset of this condition, all participants were instructed that they could quit the game at any time and enter their remaining credits into the pool of participants attempting to earn a $\$ 10$ prize for the highest score. During this optional phase, all participants were exposed to very different reinforcing contingencies on the slot machine simulation: Specifically, no slot machine options ever displayed a winning symbol combination. All spins resulted in

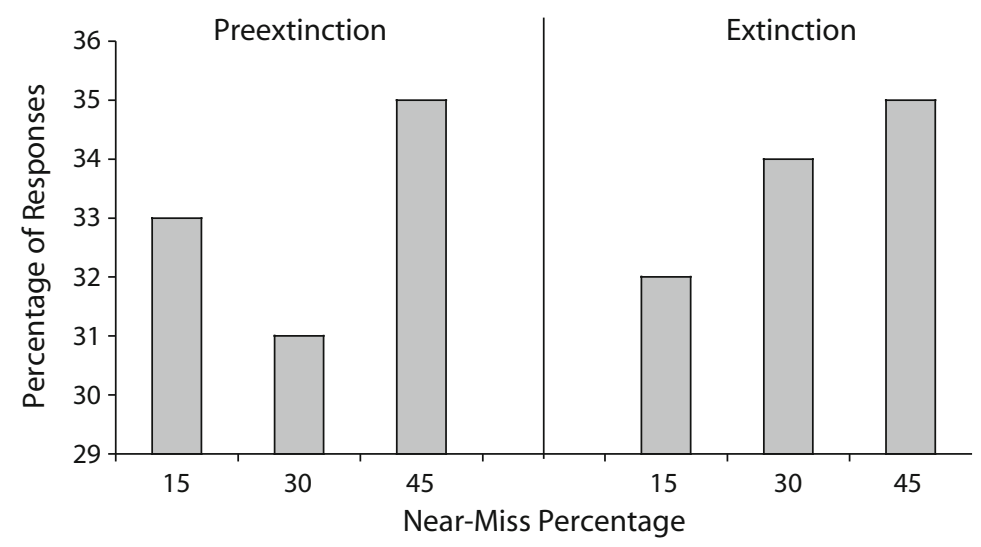

Figure 2. Mean response allocations across the three slot machine choices prior to and during extinction conditions for all participants combined. 


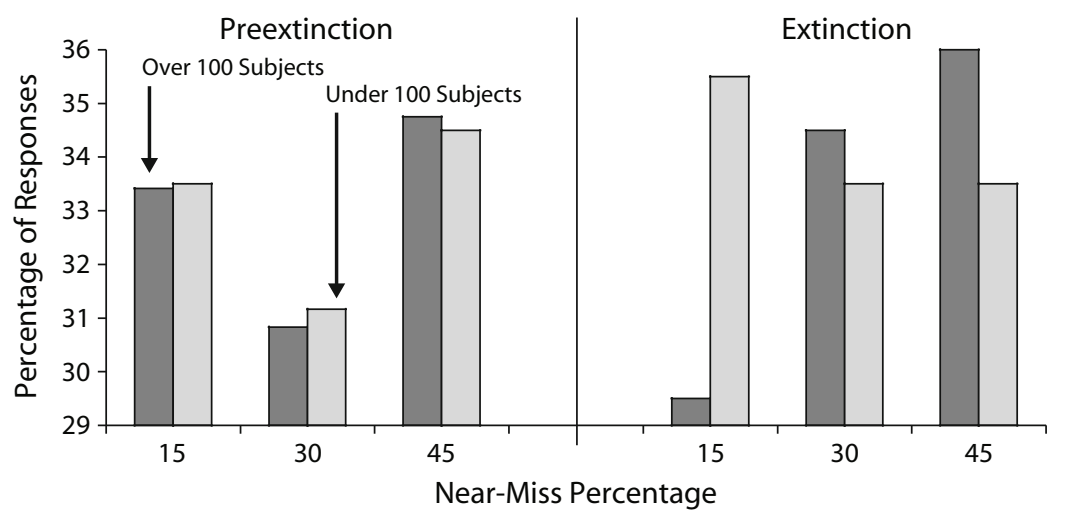

Figure 3. Mean response allocations across the three slot machine choices prior to and during extinction conditions, separated into groups of fewer than 100 optional trials played during extinction and over 100 optimal trials played during extinction.

losses; in other words, responding was placed on reinforcement extinction. Near-miss distributions continued as before, each machine displaying a given percentage of near misses on losing trials.

\section{RESULTS}

Figure 2 displays the mean percentage of responses made by the present participants across the three slot machine options. As this figure shows, there was a very slight preference across the choice options for the near-miss $45 \%$ slot machine. While this difference was slight, it was not statistically significant when all subjects were analyzed as a single group $(p<.05)$. These data suggest that, with equal reinforcement contingencies, players will generally allocate their responses across the game options. The pattern of responses was similar during both the preextinction and extinction conditions of Phase 2.

In an attempt to further understand the range of optional trials that participants played during the extinction condition of Phase 2 (range, 39-327), we qualitatively separated participants into two groups: one of more than 100 players, the other of fewer than 100 players. Twelve participants were classified as the over 100 group and 6 participants were classified as the under 100 group. These categories allowed for the analysis of players resistant to extinction conditions and of those who terminated the game during Phase 1 in fewer trials than were initially required. Figure 3 displays the mean response allocations to the various slot machine choices separated by subject group. During Phase 1's reinforcing conditions, this figure shows a similar pattern to that of Figure 2, but there is an interesting deviation during Phase 2's extinction conditions across subject group. Of specific interest is that the participants who played over 100 trials during extinction - the over 100 group - showed almost no response to the slot option containing the lowest number of near misses, and the greatest number of responses to the option containing the highest amount of near misses. A between-groups onetailed $t$ test of samples with equal variance yielded significant difference between the $15 \%$ and $45 \%$ near-miss slot machines for the over 100 group $(p<.05)$. No other analyses were significant.

Apart from preexperimental histories of gambling behavior, the only potential difference which may have resulted in a participant playing over or under 100 trials during extinction may have been the actual amount of credits won during Phase 1. Although to a considerable degree these contingencies were controlled for through the prespecification of the probability of payoff at $20 \%$ across all games, some participants might by chance have entered Phase 2 having won more or fewer credits than had other participants. Thus, the number of credits won by the over 100 and under 100 groups was examined with regard to the number of credits won at the beginning of Phase 2 or on Trial 100. Figure 4 displays the resulting analysis and indicates that, in general, participants with lower amounts of credits at the end of Phase 1 played longer during Phase 2. Perhaps these participants were chasing losses or attempting to recoup prior gains. A between-groups $t$ test was conducted comparing groups' credit totals and yielded a significant difference $(p<$ $.05)$

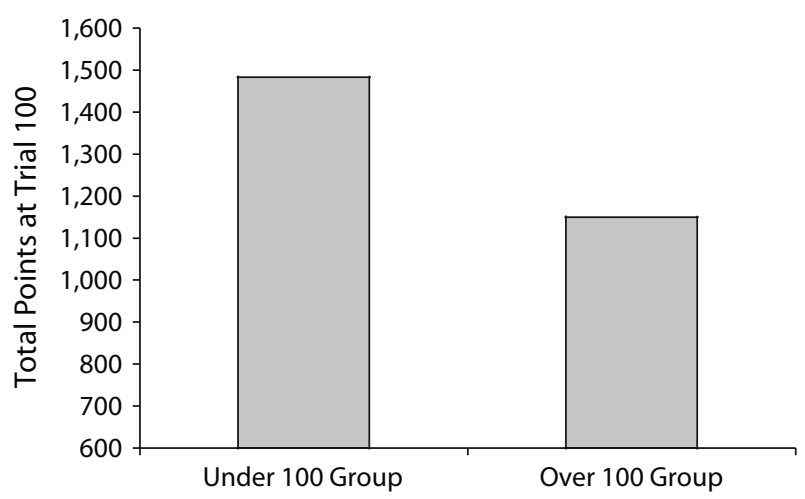

Figure 4. Total number of points earned upon entering extinction phase and the relation to whether participants played under 100 or over 100 optional trials during extinction. 


\section{DISCUSSION}

The present experiment highlights the utility of a threeoption multiple slot machine simulation for the study of gambling behavior. The present data suggest that players will generally allocate their responses equally across slot machines of equal payoff, but that when they experience a string of losses (in this case, total extinction) they may prefer the option containing the most near misses. The notion that near misses may sustain gambling behavior is not novel to the present investigation; however, the illustration that preference can be shown to exist for a near-miss game is, in fact, a new contribution to the published gambling literature.

The present data yield some interesting notions about gambling and resistance to extinction. However, the parameters of the simulation used in this study were very simplistic, and with the software described, much more research could be done on slot machine preference. For example, with near-miss distributions kept constant to assess sensitivity to reinforcement parameters, all three slot machines could yield different payback percentages. Also, rather than instating an extinction condition, the experimenter could - say, at Trial 101 - first modify the programmed contingencies, so that the machine that formerly paid off the least would now pay off the most, and then see whether the player could tell the difference. Other forms of future inquiry could assess near-miss preference at various other percentages of display than were used in the present investigation, termination of near-miss distributions during extinction, or both. With the open-ended configurations of the present software, endless opportunities exist for a researcher interested in further exploring the slot machine behavior of gamblers. Finally, pathological and nonpathological gamblers could be compared on any of the research paradigms mentioned.

In summary, gambling is a growing enterprise. It is a financial blessing for many state and local economies but a burden for those afflicted with pathological gambling disorder. Rather than rely on religion, speculation, or a moral code to dictate whether gambling is good or bad for our society, perhaps we should let science guide us. In order for it to do so, empirical investigations into the behavior of interest are needed. The software we have described is one means to that end.

\section{AUTHOR NOTE}

Address all correspondence to M. R. Dixon, Behavior Analysis and Therapy Program, Rehabilitation Institute, Southern Illinois University, Carbondale, IL 62901 (e-mail: mdixon@siu.edu).

\section{REFERENCES}

Dixon, M. R., \& Delaney, J. (2006). The impact of verbal behavior on gambling behavior. In P. M. Ghezzi, C. A. Lyons, M. R. Dixon, \& G. R. Wilson (Eds.), Gambling: Behavior theory, research, and application (pp. 171-189). Reno, NV: Context Press.

Dixon, M. R., MacLin, O. H., \& Daugherty, D. (2006). Response allocations to concurrently available slot machine simulations. Behavior Research Methods, 38, 232-236.

Dixon, M. R., \& Moore, K. (2006). Native American gambling: The quest for the new white buffalo. In P. M. Ghezzi, C. A. Lyons, M. R. Dixon, \& G. R. Wilson (Eds.), Gambling: Behavior theory, research, and application (pp. 231-247). Reno, NV: Context Press.

DiXon, M. R., \& SCHREIBER, J. E. (2004). Near-miss effects on response latencies and win estimations of slot machine players. Psychological Record, 54, 335-348.

Ghezzi, P. M., Lyons, C. A., \& Dixon, M. R. (2000). Gambling in socioeconomic perspective. In W. K. Bickel \& R. E. Vuchinich (Eds.), Reframing health behavior change with behavioral economics (pp. 313-338). Mahwah, NJ: Erlbaum.

Lesieur, H. R., \& Blume, S. B. (1987). The South Oaks Gambling Screen (SOGS): A new instrument for the identification of pathological gamblers. American Journal of Psychiatry, 144, 1184-1188.

MacLin, O. H., Dixon, M. R., \& Hayes, L. J. (1999). A computerized slot machine simulation to investigate the variables involved in gambling behavior. Behavior Research Methods, Instruments, \& Computers, 31, 731-735.

MacLin, O. H., Dixon, M. R., Robinson, A., \& Daugherty, D. (2006). Writing a simple slot machine simulation program. In P. M. Ghezzi, C. A. Lyons, M. R. Dixon, \& G. R. Wilson (Eds.), Gambling: Behavior theory, research, and application (pp. 127-154). Reno, NV: Context Press.

Shaffer, H. J., Hall, M. N., \& Vander Bilt, J. (1999). Estimating the prevalence of disordered gambling behavior in the United States and Canada: A research synthesis. American Journal of Public Health, 89, 1369-1376.

Stewart, R. M., \& Brown, R. I. F. (1988). An outcome study of Gamblers Anonymous. British Journal of Psychiatry, 152, 284-288.

(Manuscript received March 14, 2006; revision accepted for publication July 7, 2006.) 